\title{
A COMPUTATIONAL STUDY OF STEVIOL AND ITS SUGGESTED ANTICANCER ACTIVITY. A DFT AND DOCKING STUDY
}

\author{
LORENA MENESES ${ }^{1}$, SEBASTIAN CUESTA ${ }^{1}$, GUILLERMO SALGADO ${ }^{2}$, PATRICIO MUÑOZ $^{3}$, \\ ASSIA BELHASSAN ${ }^{4}$, LORENA GERLI ${ }^{5}$ AND L.H. MENDOZA-HUIZAR ${ }^{6 *}$ \\ ${ }^{1}$ Laboratorio de Quimica Computacional Facultad de Ciencias Exactas y Naturales. Pontificia Universidad Catolica del Ecuador. Ecuador, Quito. \\ ${ }^{2}$ Facultad de Ciencias Fisicas y Matemáticas, Universidad de Chile, Santiago, Chile. \\ ${ }^{3}$ Facultad de Ciencias Exactas, Departamento de Quimica. Universidad Andres Bello. Santiago, Chile. \\ ${ }^{4}$ Molecular Chemistry and Natural Substances Laboratory, Faculty of Science, Moulay Ismail University of Meknes, Morocco. \\ ${ }^{5}$ Departamento de Quimica Ambiental. Facultad de Ciencias. Universidad Catolica de la Santisima Concepcion. Concepcion, Chile. \\ ${ }^{6}$ Universidad Autónoma del Estado de Hidalgo. Academic Area of Chemistry. Carretera Pachuca-Tulancingo Km. 4.5 Mineral de la Reforma, Hgo., México.
}

\begin{abstract}
In the present, study we analyzed the electronic properties of Steviol, the Stevia rebaudiana metabolite, and its interaction with antiapoptotic protein BCL-2. The ionization potential and electrophilicity index values were evaluated in the framework of the DFT, and these values suggest that Steviol may form ligand-receptor interactions. Also, the bond dissociation energy and the electrostatic potential distribution of Steviol reveal its antioxidant behavior. Docking studies were performed to evaluate the feasibility of this molecule to interact with antiapoptotic protein BCL-2. However, no hydrogen bonds were found in the pocket site, instead six interactions, including alkyl and $\pi$-alkyl type were formed, suggesting that the possible most feasible mechanism for anticancer activity would be through free radicals scavenging.
\end{abstract}

Keywords: Steviol, BCL-2, antiapoptotic, acceptor-donor, DFT.

\section{INTRODUCTION}

Cancer has become one of the leading causes of death worldwide ${ }^{1,2}$. Some studies indicate that obesity and high caloric intake may be associated with different types of cancer including, breast, and hormone related ones ${ }^{2,3}$. The main drawback of actual oncology medication includes severe side effects, and nonspecificity on cancer cells. Therefore, the development of new therapies is needed $^{1,3}$. Literature shows that around $75 \%$ of new anticancer drugs are extracted or made from natural products ${ }^{3}$. For example, the ethanolic extract of the Stevia leaves is able to significantly inhibit the proliferation of carcinogenic cells compared to the oncology drug 5,-fluoro uracil 1 ; while its methanolic extract exhibits preventive activity and antioxidant potential against DNA strand scission by $\bullet \mathrm{OH}^{4}$. In this sense, Steviol (Figure 1) is the Stevia's major metabolite being produced as a result of the enzymatic hydrolysis done by the microflora in the intestine ${ }^{5}$. Although a study in human lymphocyte cells showed cytotoxic, mutagenic, cytotoxic effects of stevia ${ }^{6}$, several other toxicological studies show Steviol as a safe compound with low acute oral toxicity in mice ${ }^{7}$. Many other benefits of Steviol that have been reported include immunomodulatory, antiinflammatory, diuretic, anti-hypertensive, anti-hyperglycemic, antioxidant and anti-tumor activity ${ }^{3-5}$. Moreover, it has been reported that Steviol is active against gastrointestinal cancer in 6 cell types and against breast cancer in MCF-7 cells 1,3 . Steviol significantly inhibits gastrointestinal cancer cell proliferation by inducing G1 cell cycle arrest and mitochondrial apoptosis, as evidenced by the increased $\mathrm{Bax} / \mathrm{Bcl}-2$ ratio $^{1}$. Although, some studies suggest that the cell-type specificity is due to the presence of reactive oxygen species $(\mathrm{ROS})^{5}$; to the best of our knowledge, there is not reports concerning the molecular mechanisms, which explain these inhibitory effects. Thus, in this study, Steviol was characterized using quantum chemical descriptors and its possible antioxidant properties were evaluated. Also, molecular docking studies were performed to study the possible interaction and inhibition that may occur between Steviol and antiapoptotic protein BCL- $2^{8}$ to get insights about the molecular mechanism related to the Steviol anticancer activity.
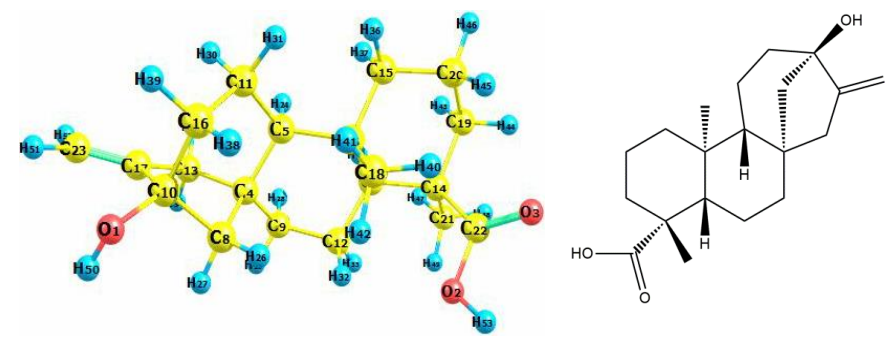

Figure 1. 2D and 3D structure of Steviol.

\section{EXPERIMENTAL}

\subsection{Computational methods}

The electronic properties of Steviol were evaluated in the framework of the density functional theory. The molecule was extracted from the Pubchem database $^{9}$ and drawn using GaussView $05^{10}$. Steviol was optimized employing the Minnesota Global Hybrid functional M062 $\mathrm{X}^{11}$, the dispersion-corrected Becke's three-parameter formulation B3LYP-D3 ${ }^{12}$, and the long-range dispersion-corrected hybrid functional $\omega \mathrm{B} 97 \mathrm{XD}{ }^{13-15}$, in combination with the triple zeta valence basis set $6-311+\mathrm{G}(\mathrm{d}, \mathrm{p})$ as implemented in Gaussian 16 program suite ${ }^{16}$. Water was chosen as solvent using the polarizable continuum model (PMC) with the solvation model density (SMD) ${ }^{17,18}$. The optimized conformers were further utilized to construct the frontier molecular orbitals and molecular electrostatic potential surfaces. Furthermore, the Highest Occupied Molecular Orbital (HOMO), and the Lowest Unoccupied Molecular Orbital (LUMO) energy values were calculated and the energy gap evaluated (equation $1)^{19}$. The global quantum mechanical descriptors used in this study were electronegativity $(\chi)$ electron affinity (EA), ionization potential (IP), chemical softness $(\sigma)$ and hardness $(\eta)$, and electrophilicity index $(\omega)$. They were calculated using equations $2-7^{19,20}$.

$$
\begin{aligned}
& \Delta \mathrm{E}=\mathrm{E}_{\text {LUMO }}-\mathrm{E}_{\text {HOMO }} \\
& \mathrm{EA}=-\mathrm{E}_{\text {LUMO }} \\
& \mathrm{IP}=-\mathrm{E}_{\text {HOMO }} \\
& \eta=\frac{\Delta E}{2} \\
& \sigma=\frac{1}{\eta} \\
& \chi=\frac{(I P+E A)}{2} \\
& \omega=\frac{\chi^{2}}{2 \eta}
\end{aligned}
$$

In order to evaluate the Steviol's antioxidant activity, a hydrogen atom was subtracted from the hydroxyl group in Steviol to evaluate the homolytic bond dissociation Gibbs free energy, at the B3LYP-D3/6-311+G(d,p) level of theory. Thus, the structure of Steviol without the hydrogen atom and that with the hydrogen atom and an unpaired number of electrons were optimized, and its free energy evaluated. Then, the bond dissociation free energy (HBD) was calculated through equation (8).

$\mathrm{HBD}=\mathrm{E}_{\text {steviol }}-\left(\mathrm{E}_{\mathrm{H}}+\mathrm{E}_{\text {deprotonated steviol }}\right)$ 
Finally, a docking study was performed between Steviol, steviolbioside, and antiapoptotic protein BCL-2. Steviolbioside was downloaded from the Drugbank database ${ }^{21}$. The receptor chosen for this study was the structure of Human antiapoptotic protein BCL-2 expressed in Escherichia coli obtained using NMR, and downloaded from the RCSB Protein Data Bank $(\mathrm{PDB}=1 \mathrm{GJH})^{8,22}$. Autodock tools ${ }^{23}$ and $\mathrm{Pymol}^{24}$ were used for the preparation of structures, which includes polar hydrogen addition, deletion of cofactors and water molecules. CB-Dock ${ }^{25}$ was first used for the binding cavity identification. Then, docking calculations were performed by using Autodock VINA ${ }^{26}$ by reducing the calculation box to the active site. Also, a default exhaustiveness, a $1 \AA$ spacing, and full ligand flexibility was set. Pymol was used for 3D visualization of the docking results and Lig plus $+{ }^{27}$ for $2 \mathrm{D}$ analysis.

\section{RESULTS AND DISCUSSION}

Frontier molecular orbitals facilitate the knowledge about electrophilic and nucleophilic properties of the molecular systems via interaction between the occupied and unoccupied molecular orbitals (MOs). Figure 2 shows the HOMO and LUMO of Steviol. The first characteristic found was that LUMO and HOMO are located in different molecular regions of the molecule. LUMO is located on the carboxylic acid while HOMO is on the hydroxyl group at the other end of the structure. This characteristic has been found in molecules with good binding properties, suggesting that this type of acceptor-donor characteristic is key for a ligand-receptor binding 28,29 .

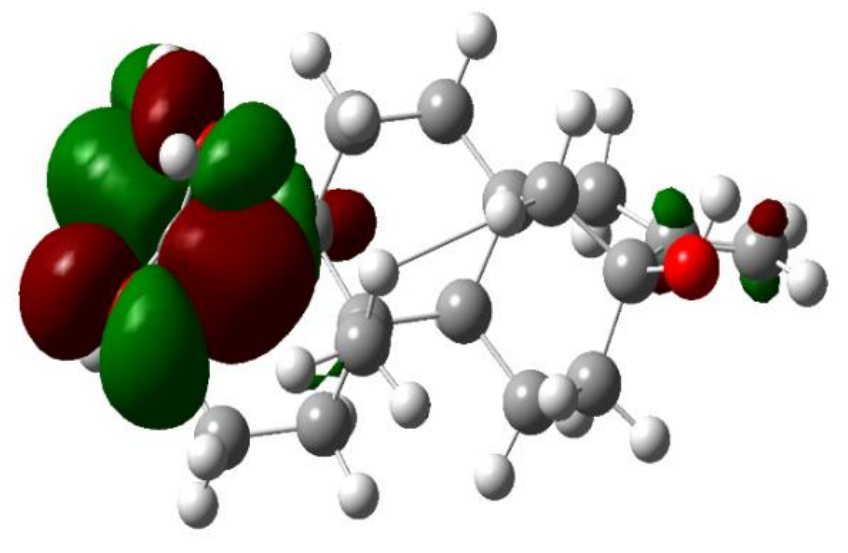

LUMO

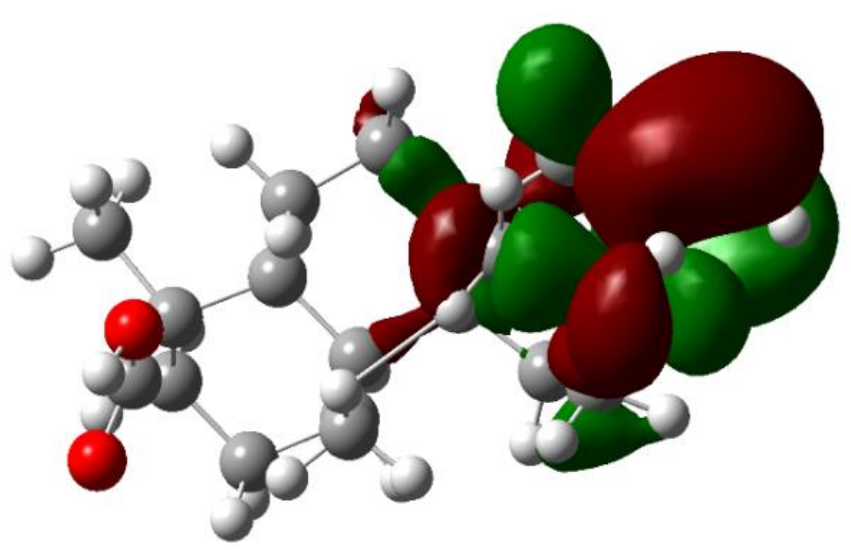

HOMO

Figure 2. Frontier Molecular Orbitals of Steviol.

Chemical reactivity of Steviol may be well understood with the help of electrostatic potential surfaces. In Figure 3, the highest electrostatic potential region is observed over the hydroxyl units present carboxylic arm and at the $\mathrm{C} 13$ position, which indicates their ability to donate electron easily to scavenge the free radicals. The oxygen atoms present in the hydroxyl unit on both sides of the molecule possess lowest electrostatic potential energy due to its valence electrons.

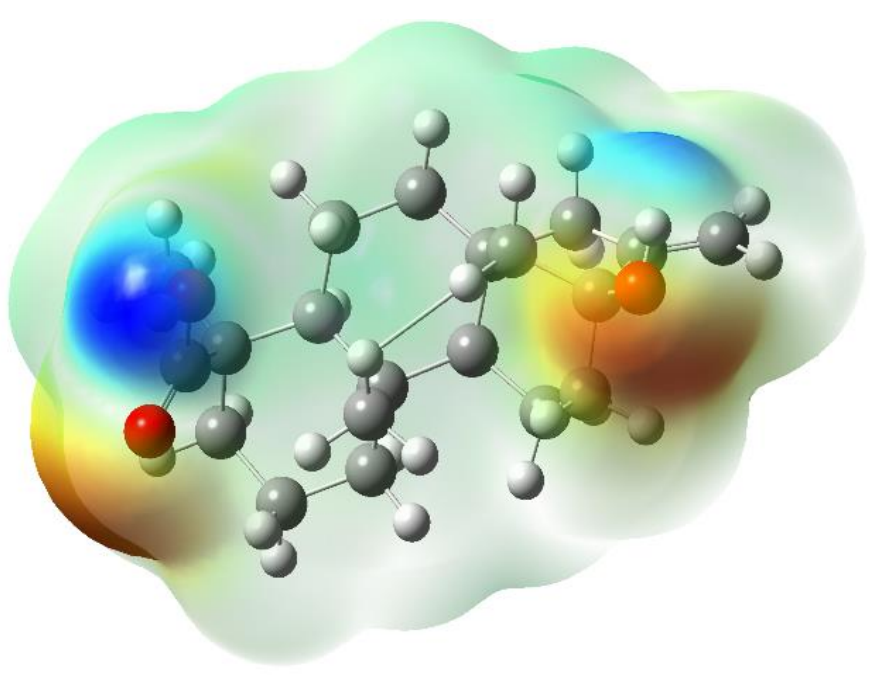

Figure 3. Electrostatic Potential surface of Steviol.

The energy gap between the HOMO and the LUMO are $6.8 \mathrm{eV}$ (B3LYP-D3), $10.6 \mathrm{eV}(\omega \mathrm{B} 97 \mathrm{XD})$ and $8.6 \mathrm{eV}(\mathrm{M} 062 \mathrm{X})$. This energy difference is due to the different dispersion correction considered in these functionals. Furthermore, as $\omega \mathrm{B} 97 \mathrm{XD}$ also consider interaction between distant atoms using a long-range empirical correction, the HOMO and LUMO energies values are usually higher.

Table 1. Quantum descriptors for Steviol in electronvolts $(\mathrm{eV})$ at the different levels of theory.

\begin{tabular}{|c|c|c|c|}
\hline & B3LYP-D3 & WB97XD & MO62X \\
\hline$H O M O$ & -6.8 & -8.9 & -8.3 \\
\hline$L U M O$ & -0.3 & 1.7 & 0.4 \\
\hline$\Delta E$ & 6.4 & 10.6 & 8.6 \\
\hline$I P$ & 6.8 & 8.9 & 8.3 \\
\hline$E A$ & 0.3 & -1.7 & -0.4 \\
\hline$\chi$ & 3.5 & 3.6 & 3.9 \\
\hline$\eta$ & 3.2 & 5.3 & 4.3 \\
\hline$\sigma$ & 0.3 & 0.2 & 0.2 \\
\hline$\omega$ & 2.0 & 1.2 & 1.8 \\
\hline
\end{tabular}

Table 1 reports the values of the global molecular descriptors calculated for Steviol using the three different functionals. From this table, it is observed that B3LYP-D3 is the only one that predicts a negative LUMO energy value, which makes a direct impact in EA being the only one positive. $\chi$ and $\sigma$ values found are similar between the three functional with values between 3.6 and $3.9 \mathrm{eV}$ for $\chi$ and around $0.2 \mathrm{eV}$ for $\sigma$. Although IP, $\mathrm{\eta}$, and, $\omega$ present differences between the three functional used, the values found suggesting good donor properties more than acceptor ones.

On the other hand, for the docking study, $\mathrm{CB}$ dock was first used to find the most feasible site of binding between BCL-2 and the two stevia compounds, Steviol and steviolbioside. Results show that from the 5 binding sites predicted, the ones with most affinity are located at different positions. This result was then compared with the crystal structure of Homo sapiens BCL-2 complexed with oncologic drug Venetoclax, and with a N-heteroaryl sulphonamide inhibitor ${ }^{30,31}$. Interestingly, none of the binding sites predicted by $\mathrm{CB}$-dock matched at the site of action of co-crystalized inhibitors (Figure 4). Therefore, the three binding sites were chosen to redock Steviol, steviolbioside, and the N-heteroaryl sulphonamide inhibitor. Table 2 shows the grid box parameters for each site, and the docking scores obtained from the docking calculation. Site 1 is N-heteroaryl sulphonamide inhibitor binding site, while site 2 and 3 are the ones found with CB-dock for steviolbioside, and Steviol respectively. 


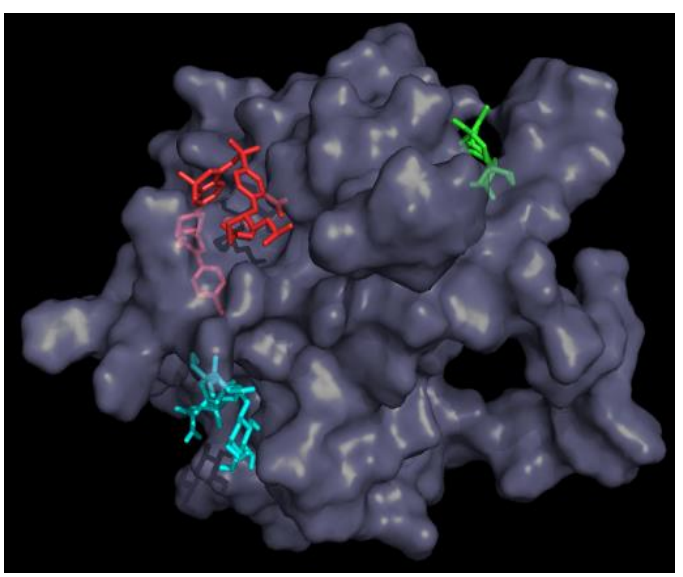

Figure 4. Best pose for the interaction of Steviol (green) and steviolbioside (cyan) with BCL-2. Co-crystalized N-heteroaryl sulphonamide inhibitor is shown in red.
Table 2. Docking scores (in $\mathrm{kcal} / \mathrm{mol}$ ) of Steviol, steviolbioside, and $\mathrm{N}$-heteroaryl sulphonamide inhibitor against BCL-2

\begin{tabular}{|c|c|c|c|c|}
\hline & Site 1 & Site 2 & Site 3 \\
\hline \multicolumn{2}{|l|}{ Steviol } & -6.7 & -5.8 & -6.4 \\
\hline \multicolumn{2}{|c|}{ Steviolbioside } & -6.8 & -7.2 & -7.7 \\
\hline \multicolumn{2}{|c|}{$\begin{array}{l}\text { N-heteroaryl sulphonamide } \\
\text { inhibitor }\end{array}$} & -7.0 & -6.3 & -6.1 \\
\hline \multirow{4}{*}{ Grid Box } & $X$ & 16.309 & 9.575 & -1.267 \\
\hline & $\mathrm{Y}$ & -0.366 & -13.439 & 14.314 \\
\hline & $\mathrm{Z}$ & 1.513 & -9.139 & 9.409 \\
\hline & Size & 20 & 20 & 20 \\
\hline
\end{tabular}

Thus, note that these results indicate that the higher binding score for Steviol and N-heteroaryl sulphonamide inhibitor is located in site 1 , while for steviolbioside in site 3 . A deep analysis of the three binding sites of steviolbioside were done in order to understand the high docking score obtained for the three sites (Figure 5).

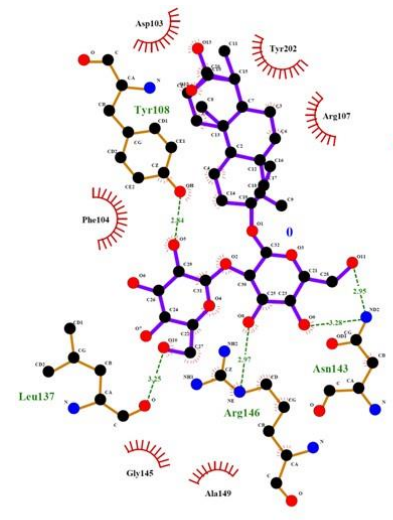

Site 1

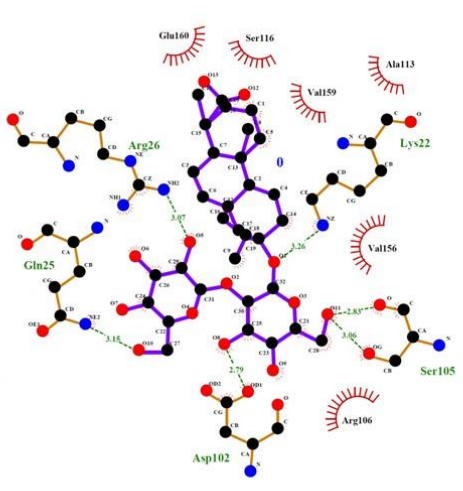

Site 2

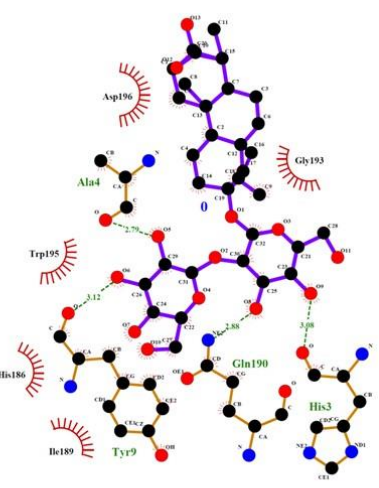

Site 3
Figure 5. Interaction of steviolbioside with the three binding sites.

Figure 5 shows that at the sites 1 and 2, steviolbioside make 10 interactions, which include 4 hydrogen bonds (HBs), at the site 3, there are 9 interactions including $4 \mathrm{HBs}$. Note that the most of these interactions occur with the glucoside portion of the molecule. This suggests that this molecule is not selective to this protein, suggesting this is not its action mechanism. Furthermore, this type of molecules are key in targeting carbohydrate receptors being able to treat viral infections ${ }^{32,33}$. If one analyzes the Steviol binding sites 1 and 3 (Figure 6) it can be seen that in site three Steviol make 9 interactions including $3 \mathrm{HBs}$. On the other hand, in the site 1, it only makes 6 interactions with no HBs. Still docking score for site one is greater than for site 3 . This suggests alkyl and $\pi$-alkyl interactions are strong enough to make a good binding between the ligand and the receptor. If the interaction is occurring in this way, then it may be possible for Steviol to inhibit BCL-2.

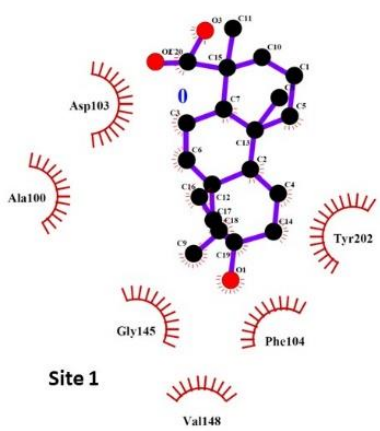

Figure 6. Interaction of Steviol with the binding sites1 and 3.

Finally, N-heteroaryl sulphonamide inhibitor was studied in order to understand the inhibition mode of BCL-2. N-heteroaryl sulphonamide inhibitor was docked to Site 1 to determine if the binding mode calculated resembles the experimental structure. Figure 7 shows the experimental structure in red and the

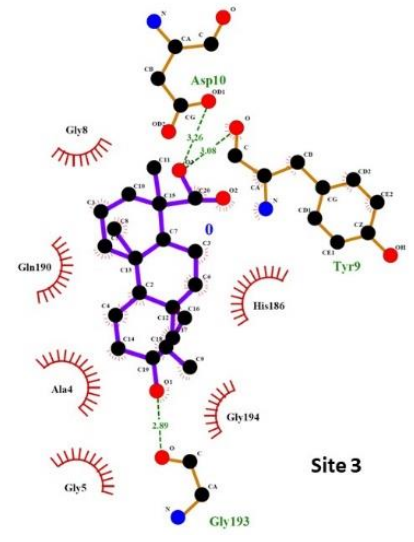

docked structure in yellow. Docking results are in agreement with the experimental one as both structures are almost overlaid in BCL-2 active pocket. Getting an insight in the interactions, N-heteroaryl sulphonamide inhibitor interacts with 11 residues forming two HBs. 

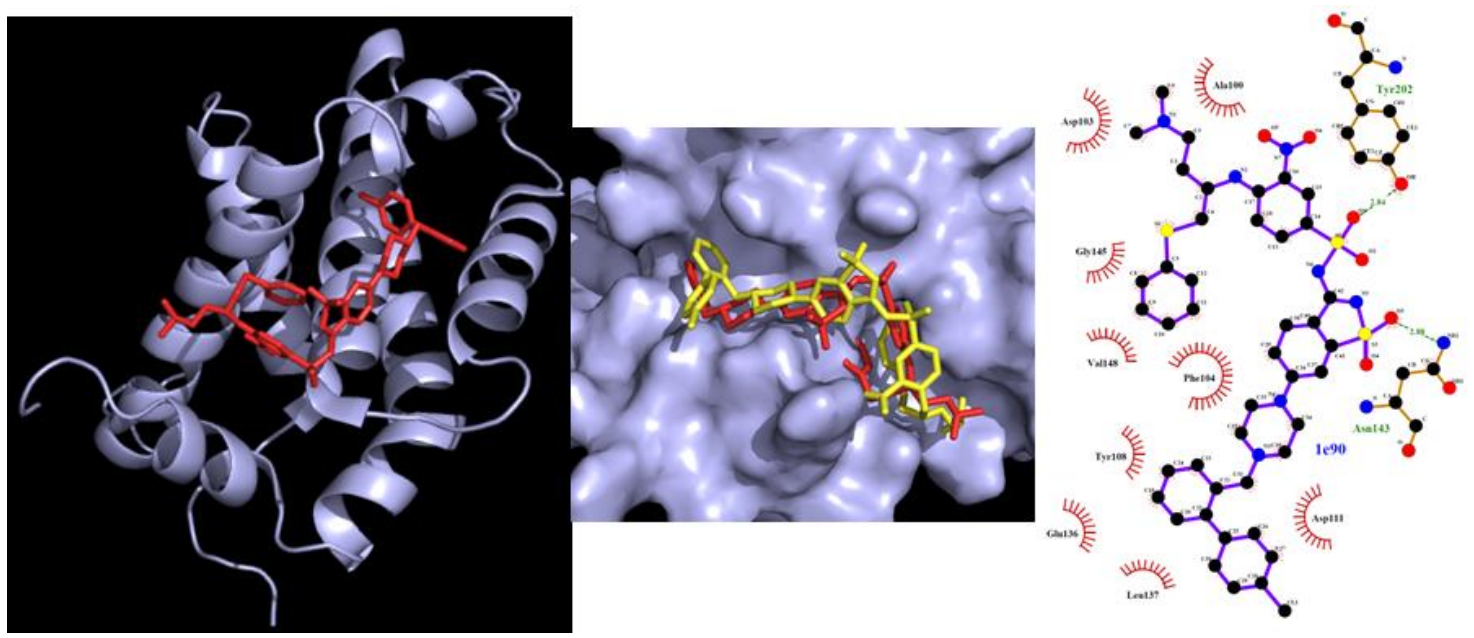

Figure 7. Interaction of BCL-2 and N-heteroaryl sulphonamide inhibitor.

\section{CONCLUSIONS}

The energy gap, ionization potential and $\omega$ values of Steviol suggest feasibility of this compound to be part of a ligand-receptor interaction. Also, the HOMO and LUMO distributions are located in opposite regions of the molecule, which has been suggested will increase the affinity in a ligand-receptor interaction. C13 is exhibiting the highest potential region, which gives the ability to donate electrons to the near hydroxyl group explaining the antioxidant behavior of Steviol. From docking studies, it was found that Steviol highest score occupies the N-heteroaryl sulphonamide inhibitor pocket indicating an inhibition may be possible. But no HBs were found, instead six interactions, including alkyl and $\pi$ alkyl type were formed, suggesting that the possible most feasible mechanism for anticancer activity would be through free radicals scavenging.

\section{ACKNOWLEDGEMENTS}

The authors gratefully acknowledge too the financial support of Direction of research UCSC, Project DIREG 03/2020, LHMH gratefully acknowledges to the Autonomous University of the State of Hidalgo.

\section{REFERENCES}

1. J. Chen, Y. Xia, X. Sui, Q. Peng, T. Zhang, J. Li, J. Zhang, Oncotarget (2018), 9, 26299-26308.

2. M. Reddy Mallu, S. Vemula, R. K. Kante. Pharm. Sci. Res. (2019), 11, 2016-2018.

https://www.jpsr.pharmainfo.in/Documents/Volumes/vol11issue05/jpsr110 51962.pdf. Accessed April 26, 2021.

3. E. Gupta, S. Kaushik, S. Purwar, R. Sharma, A. Balapure, S. Sundaram, Pharmacogn. Mag. (2017), 13, 345.

4. S. Ghanta, A. Banerjee, A. Poddar, S. Chattopadhyay, J. Agric. Food Chem. (2007), 55, 10962-10967.

5. C. Panagiotou, C. Mihailidou, G. Brauhli, O. Katsarou, P. Moutsatsou, Mol. Cell. Endocrinol. (2018), 460, 189-199.

6. T. Pasqualli, P. E. E. Chaves, c, L. da V. Pereira, É. A. Serpa, L. F. S. D. Oliveira, M. M. Machado, Immunopharmacol. Immunotoxicol. (2020), 42, 504-508.

7. D. J. Brusick, Food Chem. Toxicol. (2008) 46, S83-91.

8. A. M. Petros, A. Medek, D. G. Nettesheim, D. H. Kim, H. S. Yoon, K. Swift, E. D. Matayoshi, T. Oltersdorf, S. W. Fesik, Proc. Natl. Acad. Sci. U. S. A. (2001), 98, 3012-3017.

9. S. Kim, J. Chen, T. Cheng, A. Gindulyte, J. He, S. He, Q. Li, B. A. Shoemaker, P. A. Thiessen, B. Yu, L. Zaslavsky, J. Zhang, E. E. Bolton, Nucleic Acids Res. (2019), 47, D1102-D1109.

10. R. Dennington, T. Keith, J. Millam, Gaussview, Version 5., 2016.

11. Y. Zhao, D. G. Truhlar, Theor. Chem. Acc. (2008), 120, 215-241.

12. S. Grimme, S. Ehrlich, L. Goerigk, J. Comput. Chem. (2011), 32, 14561465

13. S. Grimme, J. Comput. Chem. (2006), 27, 1787-1799.

14. J. Da Chai, M. Head-Gordon, J. Chem. Phys. (2008), 128, 84106.

15. J. Da Chai, M. Head-Gordon, Phys. Chem. Chem. Phys. (2008), 10, 66156620.
16. M. J. Frisch, G. W. Trucks, H. B. Schlegel, G. E. Scuseria, M. A. Robb, J. R Cheeseman, G. Scalmani, V. Barone, G. A. Petersson, H. Nakatsuji, X. Li, M. Caricato, A. V Marenich, J. Bloino, B. G. Janesko, R. Gomperts, B. Mennucci, H. P. Hratchian, J. V Ortiz, A. F. Izmaylov, J. L. Sonnenberg, Williams, F. Ding, F. Lipparini, F. Egidi, J. Goings, B. Peng, A. Petrone, T. Henderson, D. Ranasinghe, V. G. Zakrzewski, J. Gao, N. Rega, G. Zheng, W. Liang, M. Hada, M. Ehara, K. Toyota, R. Fukuda, J. Hasegawa, M. Ishida, T. Nakajima, Y. Honda, O. Kitao, H. Nakai, T. Vreven, K. Throssell, J. A. Montgomery Jr., J. E. Peralta, F. Ogliaro, M. J. Bearpark, J. J. Heyd, E. N. Brothers, K. N. Kudin, V. N. Staroverov, T. A. Keith, R. Kobayashi, J. Normand, K. Raghavachari, A. P. Rendell, J. C. Burant, S. S. Iyengar, J. Tomasi, M. Cossi, J. M. Millam, M. Klene, C. Adamo, R. Cammi, J. W. Ochterski, R. L. Martin, K. Morokuma, O. Farkas, J. B. Foresman, D. J. Fox, (2016).

17. A. V. Marenich, C. J. Cramer, D. G. Truhlar, J. Phys. Chem. B (2009), 113, 6378-6396.

18. J. Tomasi, B. Mennucci, R. Cammi, Chem. Rev. (2005), 105, 2999-3093.

19. R. G. Pearson, Inorganica Chim. Acta (1992), 198-200, 781-786.

20. R. G. Parr, L. V. Szentpály, S. Liu, J. Am. Chem. Soc. (1999), 121, 1922 1924.

21. D. S. Wishart, Y. D. Feunang, A. C. Guo, E. J. Lo, A. Marcu, J. R. Grant, T. Sajed, D. Johnson, C. Li, Z. Sayeeda, N. Assempour, I. Iynkkaran, Y. Liu, A. MacIejewski, N. Gale, A. Wilson, L. Chin, R. Cummings, Di. Le, A. Pon, C. Knox, M. Wilson, Nucleic Acids Res. (2018), 46, D1074-D1082.

22. H. M. Berman, T. Battistuz, T. N. Bhat, W. F. Bluhm, P. E. Bourne, K Burkhardt, Z. Feng, G. L. Gilliland, L. Iype, S. Jain, P. Fagan, J. Marvin, D. Padilla, V. Ravichandran, B. Schneider, N. Thanki, H. Weissig, J. D. Westbrook, C. Zardecki, Acta Crystallogr. Sect. D Biol. Crystallogr. (2002), $28,235-242$.

23. G. Morris, R. Huey, W. Linkstrom, M. Sanner, R. Belew, D. Goodsell, Olson, J. Comput. Chem. (2009), 16, 2785-2791.

24. L. Schrödinger, Thomas Hold. (2015).

25. Y. Liu, M. Grimm, W. tao Dai, M. chun Hou, Z. X. Xiao, Y. Cao, Acta Pharmacol. Sin. (2020), 41, 138-144.

26. O. Trott, A. J. Olson, J. Comput. Chem. (2010), 31, 455-461.

27. R. A. Laskowski, M. B. Swindells, J. Chem. Inf. Model. (2011), 51, 2778 2786.

28. M. C. Flores, E. A. Márquez, J. R. Mora, Med. Chem. Res. (2018), 27, 844 856.

29. E. Cortes, J. R. Mora, E. Márquez, Crystals (2020), 10, 692.

30. B. B. Touré, K. Miller-Moslin, N. Yusuff, L. Perez, M. Doré, C. Joud, W. Michael, L. Dipietro, S. Van Der Plas, M. McEwan, F. Lenoir, M. Hoe, R. Karki, C. Springer, J. Sullivan, K. Levine, C. Fiorilla, X. Xie, R. Kulathila, K. Herlihy, D. Porter, M. Visser, ACS Med. Chem. Lett. (2013), 4, 186-190.

31. R. W. Birkinshaw, J. nan Gong, C. S. Luo, D. Lio, C. A. White, M. A Anderson, P. Blombery, G. Lessene, I. J. Majewski, R. Thijssen, A. W. Roberts, D. C. S. Huang, P. M. Colman, P. E. Czabotar, Nat. Commun. (2019), 10, 1-10.

32. K. Palanichamy, A. Joshi, T. Mehmetoglu-Gurbuz, M. F. Bravo, M. A Shlain, F. Schiro, Y. Naeem, H. Garg, A. B. Braunschweig, J. Med. Chem (2019), 62, 4110-4119.

33. O. Francesconi, C. Nativi, G. Gabrielli, I. De Simone, S. Noppen, J. Balzarini, S. Liekens, S. Roelens, Chem. - A Eur. J. (2015), 21, 10089-10093. 